\title{
The Indigenous Resiliency Project: a worked example of community-based participatory research
}

\author{
Julie Mooney-Somers ${ }^{\mathrm{A}, \mathrm{B}}$ and Lisa Maher ${ }^{\mathrm{A}}$ \\ ${ }^{\mathrm{A} N a t i o n a l ~ C e n t r e ~ i n ~ H I V ~ E p i d e m i o l o g y ~ a n d ~ C l i n i c a l ~ R e s e a r c h, ~}$ \\ University of New South Wales (on behalf of the Indigenous \\ Resiliency Project Australian Steering Committee) \\ ${ }^{\mathrm{B} C o r r e s p o n d i n g ~ a u t h o r . E m a i l: ~ j m o o n e y s o m e r s @ ~}$ \\ nchecr.unsw.edu.au
}

\begin{abstract}
Community-based participatory research (CBPR) is often cited as a suitable methodological approach for academic researchers wanting to work collaboratively with Indigenous communities. This paper describes the Indigenous Resiliency Project currently being conducted in Redfern, Townsville and Perth. This case study is used to demonstrate how a group of universitybased researchers and Aboriginal Community Controlled Health Services have used CBPR to work with young Indigenous Australians to explore young people's perspectives on resilience in relation to bloodborne viruses and sexually transmissible infections. This paper also describes some initial benefits gained through the process of developing the Indigenous Resiliency CBPR Project, such as: developing research capacity; establishing relationships between community organisations and research institutions; and prioritising ethical and social considerations in the conduct of research. A commentary on the experience of one health worker involved in the project accompanies the paper.
\end{abstract}

\begin{abstract}
Aboriginal people have been examined, measured and asked questions ... They have been passive subjects rather than participants. ${ }^{1}$
\end{abstract}

Despite the volume of research conducted on the health of Indigenous Australians, there is a perception that Indigenous people have derived little direct benefit from these efforts. ${ }^{2}$ The history of research on Indigenous peoples, both locally and internationally, has produced a deep suspicion of research, with a recent series of community workshops indicating that Australian Indigenous communities remain suspicious of research conducted by mainstream organisations. ${ }^{1,3,4}$ In 2002, the National Health and Medical Research Council (NHMRC) launched its Road Map, a set of guidelines for health research with Indigenous communities calling for 'community involvement in the development, conduct and communication of research'. ${ }^{5}$ More recently, the National Statement on Ethical Conduct in Human Research states that the 'research approach should value and create opportunities to draw on the knowledge and wisdom of Aboriginal and Torres Strait Islander Peoples by their active engagement in the research processes, including the interpretation of the research data' ${ }^{6}$ Aboriginal and Torres Strait Islander organisations have developed their own research protocols for researchers wanting to work with Indigenous communities, and a number of Human Research Ethics Committees have been established to assess research affecting Indigenous people and their communities. ${ }^{7}$ It is in this context that Indigenous health research increasingly involves partnerships between university-based researchers and Indigenous communities and organisations. These partnerships are seen as a way of ensuring research is responsive to community needs, conducted in a culturally appropriate manner, and beneficial to the community.

Community-based participatory research (CBPR) is an approach that allows researchers to work with communities to generate knowledge about and solutions to problems the community is facing. This framework repositions the people who would usually be the object of the research as participants in the research process; 'the researched become the researchers' ${ }^{8}{ }^{8}$ CBPR involves more than consultation; it focuses on developing community capacity to participate as co-investigators in developing, conducting and disseminating the research. ${ }^{9}$ It encompasses approaches such as participatory action research, action research, partnership research and collaborative inquiry, and is characterised by an emphasis on communities as coresearchers. A review of CBPR undertaken in the United States suggests two core elements: a reciprocal co-learner relationship between researchers and communities (which includes shared decision-making and the removal of barriers to participation); and the immediate and direct benefit 
of new knowledge (which includes shared ownership of research products). ${ }^{9}$

CBPR has often been utilised in research with vulnerable or marginalised populations and is increasingly employed in research with Indigenous communities. ${ }^{1,9-17}$ The principles and characteristics of CBPR are considered to have the potential to address the failings, and 'colonisingeffects', of previous research on Indigenous peoples. ${ }^{8}$ Further, by involving affected communities in the analysis and interpretation of data, CBPR has the potential to avoid the misrepresentation of 'Indigenous societies, culture and persons by non-Indigenous academics and professionals'. Instead of seeing 'experts' - usually non-Indigenous people - as the only legitimate source of knowledge, CBPR recognises and values the knowledge of 'ordinary' people. While the technical knowledge of researchers is valuable, it is not the only legitimate way of knowing about the world. ${ }^{18}$ Moreover, prioritising community members' knowledge of community needs and perspectives may increase the likelihood of any intervention arising from the research having beneficial outcomes for the affected community. ${ }^{9}$ While a CBPR framework is increasingly used in mainstream public health research, there are few published examples of the day-to-day practicalities of using this framework to undertake research with Indigenous communities in Australia. This paper uses the Indigenous Resiliency Project as a case study to demonstrate how a CBPR approach can be employed to develop communitybased research into highly sensitive and challenging health issues.

\section{The Indigenous Resiliency Project}

The Indigenous Resiliency CBPR Project is one component of an international collaboration exploring the role of resiliency in responding to bloodborne viruses (BBVs) and sexually transmissible infections (STIs) in Indigenous communities in Australia, New Zealand and Canada. Funded by the NHMRC, the Australian component is being undertaken by a collaboration of Aboriginal Community Controlled Health Services (ACCHS): Townsville Aboriginal and Islanders Health Service (TAIHS), Aboriginal Medical Service, Redfern (AMS Redfern) and Derbarl Yerrigan Health Service, Perth (DY); a research institution, the National Centre in HIV Epidemiology and Clinical Research; and several independent Indigenous researchers. This collaboration worked with international partners to develop a funding application. The Boards of Directors of the three participating ACCHS reviewed and approved each component of the project during the initial project development stage, and again when each component began. Formal ethical review processes have been followed through the Human Research Ethics Committees of the Aboriginal Health and Medical Research Council of NSW, the Western Australian Office of Aboriginal Health and the University of New South Wales. An Australian Steering Committee (ASC), made up of two representatives from the three community partners and the research partner, along with several independent Indigenous researchers, oversees the development of the project. The ASC provides guidance on scientific, administrative and budgetary matters and determines areas of priority for the project. The ASC plays a vital leadership role in advising on cultural matters related to the conduct of the study, including the review and approval of all project dissemination, and assists in strengthening communication with all key stakeholder communities. This is the forum through which shared decision-making is achieved and the shared ownership of research products is protected. ${ }^{9}$

The Indigenous Resiliency CBPR Project brings young Indigenous Australians, participating health services and university-based researchers together to develop and conduct qualitative research on what protects young Indigenous Australians against BBVs and STIs. It aims to build the capacity of participating health services in research practice; identify, assess and enhance the STI and BBV resilience capability of Aboriginal and Torres Strait Islander people in three project sites; and inform opportunities to decrease the risk of STI and BBV transmission in project site communities. In each project site, a locally employed (but centrally-funded) site coordinator and health service staff, under the guidance of their Board of Directors, work with a project-based qualitative study coordinator and university-based researchers to engage with young Indigenous people from the local community (peer researchers) to develop and conduct the project. The Indigenous Resiliency CBPR Project is not a multi-site project where the same protocol is implemented across all sites. Instead, each project 'is a custom job', with the local projects recognising diversity by developing in response to the priorities - and capacities - of the local community and health service. ${ }^{19}$

\section{Methods}

Individual interviews and focus groups are being used to explore young people's lived experiences of sexual behaviour and drug use, learning about STIs and BBVs in their families and communities, and accessing services for prevention, testing and treatment. These qualitative approaches offer opportunities for understanding the meaning of sexual behaviour and drug use, and the contexts in which people contract or avoid STIs and BBVs. Individual face-to-face interviews, in particular, allow peer researchers to create a space where participants can share stories of how they have drawn on their own and their community's strengths to keep themselves protected against STIs and BBVs.

CBPR is a dynamic process, with the project emerging as the process proceeds. Project questions and processes are 
likely to be progressively redefined and qualitative methods are conducive to this. It is also necessary for the project to be flexible to the developing skill and confidence of peer researchers (and health service staff). In CBPR, methods are selected on the basis that they are 'useful and useable to all those participating in the process'. ${ }^{18}$ This means selecting methods that can be taught quickly to people with a broad range of educational experiences and literacy skills. To date, 20 young people and many health service staff and mentors have been trained in qualitative sampling, developing interview questions, and conducting and recording interviews. In due course, these young people, health service staff and mentors will participate in the thematic analysis of the qualitative data they have collected and disseminate findings through the preparation of community reports and involvement in community forums. People have been provided with the skills required to participate actively in each stage of the research process. The methods used do not need expensive hardware or software. Indeed, most of the data has been collected using pen and paper. This has allowed the development of a skill base that is more likely to be sustainable when the project ceases.

The relationship between interviewer and interviewee is crucial in qualitative methods as data is generated through their interactions. The Indigenous Resiliency CBPR Project concerns personal and often stigmatised behaviours, and the research teams spent a lot of time discussing how to talk about these in sensitive and culturally appropriate ways. Semi-structured interviews and focus groups allow peer researchers to use their knowledge and expertise to conduct a conversation around the interview questions and adapt their questions and style for individual participants. Interviews usually began with a conversation about where the participants' and researchers' families were from. This was an important cultural protocol to follow and the amount of time spent on this varied considerably, especially if there was a shared family connection. The first question in the interview schedule (in both Townsville and Redfern) was about the participant's history. This allowed the participant to provide a context for the interview, and for the researcher and participant to get to know each other before questions about STIs and BBVs began. Again, this was an important cultural protocol but is not usual for a qualitative research interview.

CBPR recognises local methods of knowledge gathering as valid. ${ }^{19}$ Semi-structured interview schedules were developed in the research training and development workshop held at each site. This meant that the peer researchers and health service staff shaped the question content and the way questions were to be asked. Interviewers used the schedule to guide their conversation, encouraging participants to share personal experiences and stories from their lives. Data was collected by spending time meeting and getting to know people in the community (potential participants and others). Individual interviews were conducted on the street, in parks and shopping malls and in community-owned spaces. The flexibility of a qualitative approach meant that interviews could be held when an opportunity arose, and could, if necessary, be started, paused and resumed at a different time. In the two active project sites, 95 individual interviews and seven focus groups have been conducted with young Indigenous Australians. In each case, an Indigenous researcher - peer, health service staff or mentor - was the interviewer or focus group facilitator.

At the time of writing, one project site is engaged in data analysis, a second is actively involved in data collection, and the third is developing the project, so there are no outcome data to report. However, the aims of the project are not exclusively oriented to data-driven outcomes. CBPR foregrounds action and changes occur in service provision and the lives of people participating in the research throughout the project. ${ }^{9}$ The remainder of this paper will reflect on the process of the research and describe some process-driven outcomes.

\section{Discussion}

\section{Reflecting community priorities}

The ASC asked each health service to identify a priority population to engage. In TAIHS, a consultation process was undertaken internally and with key stakeholders and community members. Consensus emerged around homeless and residentially unstable young people as the priority population group. ${ }^{20}$ In contrast, the AMS Redfern had an existing association with a local Aboriginal Men's Group (Babana), whom they invited to become a partner in the local project. Thus, their priority population became young men.

The university-based researchers developed a three-day research development and training workshop covering research ethics, communication, research sampling and recruitment, individual and group interviewing, participant observation, writing field notes and analysis of qualitative data (these workshops are described elsewhere). ${ }^{21}$ Each workshop was tailored to the relevant priority population and the educational level of the peer researchers taking part in the project. Peer researchers, health services staff and - in Redfern - mentors, participated in the workshops. Over the course of the workshops, research processes and materials fundamental to the project were developed. These included inclusion and exclusion criteria for research participants; a recruitment plan; a list of topics and questions for the individual and group interviews; and introductory statements for interviews. In this way, peer researchers, health service staff and universitybased researchers collaboratively developed project questions reflecting local priorities and meanings, and 
established ways of conducting the project that were appropriate to the priority population and the local community. ${ }^{20,21}$

\section{Working with peer researchers}

At the heart of CBPR is a commitment to work with people affected by the issue under investigation. For the Indigenous Resiliency CBPR Project, this means inviting young Indigenous people from the local community to engage with the project as peer researchers - from the development of research questions and materials, to data collection, data analysis and dissemination. It also means valuing the knowledge peer researchers have gained through their lived experiences, and giving them a mandate to influence the project and its process.

In Townsville and Redfern, the journeys of the peer researchers have been quite different. ${ }^{20}$ In Townsville, eight young men and women who had experienced residential insecurity (either personally or through a close family member or friend) were recruited through local contacts and health promotion events. These young people participated in the research training and development workshop and four were invited to work with the local team to develop and conduct the project. By the focus group stage, most of the original peer researchers were no longer involved and a ninth young person was trained to participate in the final stage of data collection. The AMS Redfern, in partnership with Babana Aboriginal Men's Group, identified eight young men through professional or community contacts. All eight were asked to make a commitment to engage with the project for the duration ( 2 days per week for 4 months). The AMS Redfern and Babana Aboriginal Men's Group introduced a parallel mentoring program where members of the men's group were matched with peer researchers to provide ongoing cultural support. This unique and valuable innovation has undoubtedly been crucial in maintaining the level of peer researcher involvement, with eight peers involved throughout. In keeping with the spirit of CBPR, the role of these mentors evolved during the project and they became an integral part of the research, attending research meetings and accompanying peer researchers during field work and data collection.

There has been a lot of discussion within the project teams about changes in the young people engaged as researchers. Health service staff and mentors have commented on their increasing self-esteem and confidence, and their willingness to speak out about issues they feel the project needs to address differently. The young people themselves have spoken of an increased sense of community belonging gained from working within a community organisation. The project has increased the youth voices within the participating health services, creating opportunities for dialogue between young people and health workers. Being involved in the project has also connected the participants to people who have become invested in their future. Opportunities for training and employment have been regularly brought to the peers' attention. Two of the peer researchers are now working in health service delivery within community-controlled and mainstream organisations. Important connections are also being made between current leaders in the local Indigenous community and the peer researchers, themselves potential community leaders. Several of the peer researchers have been invited to sit as community or youth representatives on advisory boards and committees.

\section{Research as action}

The Indigenous Resiliency CBPR Project has had quite a high profile within the participating health services. For the duration of the project, there are dedicated site coordinators based within each health service and other staff who work closely with the project. In Redfern, eight peer researchers, eight mentors, two health service staff and a university-based researcher worked in the health service 2 days a week for 4 months. This is a significant presence, and raises the profile not only of the Indigenous Resiliency CBPR Project but also of research generally. The health service's support of research is demonstrated to staff, clients and other people visiting the service. More importantly, a research skill base is being developed within the health service, and in the case of the peer researchers (and in Redfern, the mentors), in the local community. This is an important outcome, and addresses the first aim of the Indigenous Resiliency CBPR Project to develop research capacity. It is too early to judge the success or sustainability of this capacity; however, in both health services conversations have begun about future programs of research, some involving a CBPR framework.

The research teams spend a significant amount of time in the community recruiting participants and collecting data. The peer researchers, site coordinators, and, in Redfern, the mentors, wear identification badges and introduce themselves as conducting a research project with TAIHS or the AMS Redfern. This is beneficial to the project as the research is vouched for by a respected community organisation. There is also an important benefit to the community, as the research teams are effectively promoting a community-controlled health service, often to people who are considered 'hard to reach'. In Townsville in particular, some of the more mobile young people were not aware of TAIHS and were keen to get more information about a health service run by and for their community. In both sites, people often asked about accessing the health service or sought help with making appointments. Whilst the Indigenous Resiliency CBPR Project is not a peer education project, there is a strong desire among the peer researchers to provide information to people who participate in the 
project. The project formally supported the principle of 'no research without service' by organising referrals and having information available for peer researchers to distribute. More informally, peer researchers, health service staff and mentors spoke to many people (who did not necessarily participate in the project) about STIs and BBVs. Having members of the community talking openly about STIs and BBVs raises the profile of these infections and may help alleviate the shame associated with discussing them.

A less predictable outcome of the project has been th connections established between the health services and external organisations that provide services to the priority population in each project site. In Townsville, a service that provided a venue for a focus group asked for regular discussions around sexual and other health issues to be provided for the young people attending their service. During the first AMS Redfern workshop, participants visited several organisations that provide services to young people. The mentors (who include staff from local high schools and the probation and parole service) and health service staff made contact with programs that could be useful to their clients. Similarly, the mainstream organisations made contact with their local communitycontrolled health service, a connection that could facilitate future referrals for their Indigenous clients.

\section{Conclusion}

Until Indigenous communities have the resources or capacity to conduct their own research, partnerships with university-based researchers who bring technical expertise are inevitable. ${ }^{13}$ CBPR is an approach that simultaneously facilitates a research partnership and provides 'the training and resources that will allow the community to act on its own behalf in the future' ${ }^{18}$

This paper has described how the Indigenous Resiliency Project has utilised a CBPR approach to build a partnership between community organisations and research institutions, and to develop a project that is communityowned, locally relevant and culturally appropriate. An early benefit of this approach, and a key objective of the project, is that community members have been trained to conduct research that responds to the health priorities set by their communities. It is too early to evaluate the long-term sustainability or success of this research capacity, which may include seeking research funding, creating research jobs, developing research partnerships and continuing to conduct research. ${ }^{9}$ Nor can we assess whether the relationships between the Indigenous communities, ACCHS and research institutions that have facilitated this genuinely participatory project endure and generate new projects. The principal issue that this paper cannot address at this early stage is the effect that this project will have on the health of the participating communities.
Adopting a CBPR approach is not easy; it brings considerable challenges for researchers and communities. CBPR requires considerably more time, money, personnel and personal commitment than traditional research approaches. As Baum and colleagues note, it is messy, unpredictable and there are often differences in priorities between researchers and communities. ${ }^{8}$ However, unlike other research approaches, CBPR combines research and action in a way that has the potential for communities to see the benefits of research conducted by, rather than on, them.

\section{Acknowledgments}

This paper is written on behalf of the Indigenous Resiliency Project Australian Steering Committee: Angie Akee and Robert Scott (Townsville Aboriginal and Islanders Health Service), John Daniels and Dulcie Flowers (Aboriginal Medical Service, Redfern), Colin Garlett (Derbarl Yerrigan Health Service), John Kaldor and Lisa Maher (National Centre in HIV Epidemiology and Clinical Research) and independent investigators Sandra Eades, Chris Lawrence, Maurice Shipp and Edward Wilkes. As per our project protocols, this paper was circulated to members of the ASC for review and approval. Where internal protocols dictated, ASC members also circulated the paper to their health service Board of Directors, management and relevant staff. We acknowledge the contributions of David Brockman, the project's national coordinator until April 2008; Wani Erick, the Townsville Aboriginal and Islanders Health Service site coordinator until December 2008; and John Williams, the current site coordinator at the Aboriginal Medical Service, Redfern. We thank the project staff, peer researchers, mentors and participants for their many contributions.

The Indigenous Resiliency Project is funded by the International Collaboration in Indigenous Health Research Program, a trilateral partnership between the National Health and Medical Research Council of Australia, the Canadian Institutes of Health Research, and the Health Research Council of New Zealand. The National Centre in HIV Epidemiology and Clinical Research is core-funded by the Australian Government Department of Health and Ageing, and Lisa Maher is supported by the award of an NHMRC Research Fellowship.

\section{References}

1. Holmes W, Stewart P, Garrow A, Anderson I, Thorpe L. Researching Aboriginal health: experience from a study of urban young people's health and well-being. Soc Sci Med 2002; 54(8): 1267-79. doi:10.1016/S0277-9536(01)00095-8

2. Cochran PA, Marshall CA, Garcia-Downing C, Kendall E, Cook D, McCubbin L et al. Indigenous ways of knowing: implications for participatory research and community. Am J Public Health 2008; 98(1): 22-7. doi:10.2105/ AJPH.2006.093641

3. Smith LT. Decolonizing methodologies: research and indigenous peoples. London, New York: Zed Books; 1999.

4. Onemda VicHealth Koori Health Unit. We can like research ... in Koori hands: A community report on Onemda VicHealth Koori Health Unit's research workshops in 2007. Melbourne: The University of Melbourne; 2008.

5. National Health and Medical Research Council. The NHMRC Road Map: A Strategic Framework for Improving Aboriginal and Torres Strait Islander Health through Research. Canberra: National Health and Medical Research Council; 2002. 
6. National Health and Medical Research Council, Australian Research Council, Australian Vice-Chancellors' Committee. National Statement on Ethical Conduct in Human Research. Canberra: National Health and Medical Research Council; 2007.

7. Aboriginal Health and Medical Research Council of New South Wales. AH\&MRC Guidelines for Research into Aboriginal Health: Key principles. Sydney: Aboriginal Health and Medical Research Council of New South Wales; 2008.

8. Baum F, MacDougall C, Smith D. Participatory action research. J Epidemiol Community Health 2006; 60: 854-7. doi:10.1136/jech.2004.028662

9. Viswanathan M, Ammerman A, Eng E, Garlehner G, Lohr K, Griffith D et al. Community-based participatory research: assessing the evidence. Rockville, MD: Agency for Healthcare Research and Quality; 2004.

10. Coupland H, Maher L. Clients or colleagues? Reflections on the process of participatory action research with young injecting drug users. Int J Drug Policy 2005; 16(3): 191-8. doi:10.1016/j.drugpo.2004.12.002

11. Bostock J, Freeman J. 'No limits': Doing participatory action research with young people in Northumberland. J Community Appl Soc Psychol 2003; 13(6): 464-74. doi:10.1002/casp.751

12. Nelson G, Ochocka J, Griffin K, Lord J. 'Nothing about me, without me': participatory action research with selfhelp/mutual aid organizations for psychiatric consumers/ survivors. Am J Community Psychol 1998; 26(6): 881-912. doi:10.1023/A:1022298129812

13. Pyett $P$. Working together to reduce health inequalities: reflections on a collaborative participatory approach to health research. Aust N Z J Public Health 2002; 26(4): 332-6. doi:10.1111/j.1467-842X.2002.tb00180.x

14. Tsey K, Patterson D, Whiteside M, Baird L, Baird B, Tsey K. A microanalysis of a participatory action research process with a rural Aboriginal men's health group. Australian Journal of Primary Health 2004; 10(1): 64-71.
15. Hecker R, Gordon E, Delaney S, Schuil C, Capon A, editors. The benefits of participatory evaluation methods in evaluating Aboriginal health programs. Evaluation: Equipping communities and government conference; 1997; Curtin, ACT. Australasian Evaluation Society.

16. Larkins SL, Page RP, Panaretto KS, Scott R, Mitchell MR, Alberts $\mathrm{V}$ et al. Attitudes and behaviours of young Indigenous people in Townsville concerning relationships, sex and contraception: the "U Mob Yarn Up" project. Med J Aust 2007; 186(10): 513-8.

17. Brough M, Bond C, Hunt J, Jenkins D, Shannon C, Schubert L. Social capital meets identity: Aboriginality in an urban setting. J Sociol (Melb) 2006; 42(4): 396-411. doi:10.1177/ 1440783306069996

18. Brydon-Miller M. Using Participatory Action Research to Address Community Health Issues. In: Murray M, editor. Critical Health Psychology. London: Palgrave; 2003, pp. 187-202.

19. Kidd SA, Kral MJ. Practicing participatory action research. J Couns Psychol 2005; 52(2): 187-95. doi:10.1037/00220167.52.2.187

20. Erick W, Mooney-Somers J, Akee A, Maher L. Resilience to blood borne and sexually transmitted infections: the development of a participatory action research project with young Aboriginal and Torres Strait Islander people in Townsville. Aborig Isl Health Work J 2008; 32(6): 5-8.

21. Mooney-Somers J, Erick W, Brockman D, Scott R, Maher L. Indigenous Resiliency Project Participatory Action Research Component: A report on the Research Training and Development Workshop, Townsville, February 2008. Sydney: National Centre in HIV Epidemiology and Clinical Research, The University of New South Wales; 2008. Available from: http://www.nchecr.unsw.edu.au/NCHECRweb.nsf/resources/ PAR-report/\$file/PARwshop-report_TAIHS.pdf (Cited 3 July 2009.) 


\section{Commentary}

Robert Scott is the men's sexual health worker at the Townsville Aboriginal and Islanders Health Service (TAIHS). He has been actively involved in the Indigenous Resiliency Project. This is a reflection on his involvement.

I remember when the researchers from Sydney made their first visit to our service (TAIHS) to talk about the project. The staff didn't think the project would have much to do with us as it seemed to be all about management and the timeline was quite long. I became involved because of my job as a sexual health worker and sat in on the teleconferences. It was like going from high school to a university lecture. There was a lot of information and background and often it was hard to understand. When I went to my first face-to-face meeting in Perth, the picture became much clearer and because I was away from work I could spend time and talk to people. I asked a lot of questions and got a good insight into the project.

In the past, research has not always been done properly, and this has caused community unease. It was hard when I got back to work because there was a lot of resistance in the service and the community to research. Our clinic staff did not understand what the project was about and put stickers on the front cover of peoples' charts that read, 'The contents of this file is not to be used for research'. This was a complete misunderstanding as we were never going to access charts but it shows how important it is to consult with people properly before you begin.

When the project started, we talked about the kind of personnel we needed. I was worried that if we had a health worker in the coordinator position they would end up doing non-project work. In the end, we did employ a registered nurse (Wani Erick). Sometimes the health service has needed Wani's and my clinical skills more than they have needed the project to progress. The tension between research and service delivery is a familiar one for Aboriginal and other health workers.

The only thing I think we could have done better was to have a male worker more available to do some of the interviews with young men. I was on clinic duty during the data collection time and was unable to help much with that aspect, so we have a bias toward women in our sample.

The project has had an impact on the TAIHS workforce. The research has made people more talkative and that has the potential to improve community knowledge. Wani and I shared a lot of information about STIs and BBVs that has been passed on to our clients. The fieldwork really raised awareness of our service in the community, especially among young people at risk who didn't access our service. Wani got a lot of requests for information, services and referrals and because she was based in the service she knew all the doctors here, when they worked and what services people could access, so she could connect them directly to the right things and organise appointments or transport.

At the beginning of the project, we used a decision matrix to identify the target population our service was going to work with. Working through the criteria in the matrix was a good way to inform people about the project, because we talked to lots of people in the service and the community. TAIHS has a very successful mothers and babies clinic so the obvious population for us was pregnant women, but in some ways that was too easy. Thinking about who would benefit from the research and who needed it meant we selected residentially insecure young people. We provide some services to this population already but this was a concentrated way to engage them and give something back. A comfortable and secure home life is so important for young people and I see a lot coming through here that have problems at home or are living in overcrowded houses. Having this documented through the research is a very positive outcome. It shows our strengths and our weakness as a service, and that young people are concerned about their health in different ways. I hope we can use this to apply for extra funding because the only real measure of success is seeing actual changes. 